it, increasing the chance for ions to permeate. On the other hand, the ion concentration in the central cavity saturates at high concentration in the bulk, and the chance for ions to enter into the selectivity filter becomes constant, resulting in the saturation of ion permeation. Thus, the saturation of the ion concentration in the central cavity makes the Michaelis-Menten behavior of the conductance-concentration curve.

\section{A1148 カリウムチャネルのイオン透過をイオン一水分子列で可視化する}

Envisioning the ion permeation through the KesA potassium channel as queues of ion and water arrays

Masayuki Iwamoto, Shigetoshi Oiki (University of Fukui Faculty of Medical Sciences)

The ion distribution in the crystal structure of the KcsA potassium channel dramatically advanced our understanding of the mechanism of ion permeation. These still pictures under equilibrium conditions, however, may not be a snapshot of on-going permeation processes. To understand dynamics of permeation, here we counted the ratios of ion and water flow (the water-ion coupling ratio: $\left.C R_{w-i}\right)$ through the KcsA channel by measuring the streaming potential $\left(V_{\text {stream }}\right)$. Water flow driven by the osmotic pressure flushes out ions in the narrow selectivity filter and generates potential differences across the membrane $\left(V_{\text {stream }}\right)$. The $V_{\text {stream }}$ value was converted to the $C R_{w-i}$ value, which tells us how individual ion and water molecules are queued in the narrow and short filter during permeation. At high $\mathrm{K}^{+}$concentrations, the $C R_{w-i}$ value was 1.0 , indicating that turnovers between the alternative ion and water arrays in a single-file undergoes. At low $\mathrm{K}^{+}$, the $C R_{w-i}$ value was increased over 2.2, suggesting that the filter contained mostly one ion at a time. These average behaviors of permeation were kinetically analyzed for more detailed permeating processes. Here we envisioned the permeation as queues of ion and water and sequential transitions between different patterns of arrays. At physiological conditions, we predicted that the "knock-on" mechanism may not be predominant.

\section{3:10 16:10 A 会場 / Room A 生体膜・人工膜 4 (輸送、情報伝達) \\ Biol \& Artifi memb 4: Transport, Signal transduction}

\section{A1322 Effects of positive charges on the movement of polypeptide chain through the ER translocon}

Hidenobu Fujita, Yuichiro Kida, Marifu Yamagishi, Masao Sakaguchi (Grad Sch. Sci., Univ. Hyogo)

In eukaryotic cells, polypeptide chains are co-translationaly translocated through and integrated into the ER membrane via protein translocation channel, translocon. Positively charged amino acid residues are well recognized as topology determinants of membrane proteins, and found predominantly on the cytoplasmic side of the membrane. Consistent with this, positively charged residues in immediate neighborhood of hydrophobic segment contribute to stoptranslocation of polypeptide trnaslocating through the translocon. Here we investigated effect of positive charges on the polypeptide chain movement through the translocon and, surprisingly, found that the positive charges facilitated membrane spanning of a marginally hydrophobic segment, even when separated by 70 residues from the segment. In this case, the segment is exposed to the lumen and then the downstream positive charges trigger the segment to slide back into the membrane. Moreover we found that a cluster of positively charged residues arrest translocation, while negatively charged residues are not. Negative charges inserted into the positive charge cluste negate the translocation-arrest, and high salt conditions also suppress it. We concluded that positive charges arrest the polypeptide movement via simple electrostatic interaction. The positive charge cluster causes the translocating nascent chain to fluctuate back and forth. The positive charges not only fix the hydrophobic segment in the membrane, but also exert a much more dynamic action than previously realized.

\section{$3 A 1334$ シリコン基板上微小井戸アレイによる} ルシウムイオン透過計測

$\mathrm{Ca}^{2+}$ ion transport through $\alpha$-hemolysin channels monitored by microwell array on $\mathrm{Si}$ substrate

Koji Sumitomo', Arianna McAllister ${ }^{1}$, Yukihiro Tamba ${ }^{2}$, Yoshiaki Kashimura ${ }^{1}$, Aya Tanaka ${ }^{1}$, Youichi Shinozaki ${ }^{1}$, Keiichi Torimitsu ${ }^{1}$ ( ${ }^{1}$ NTT Basic Research Labs., ${ }^{2}$ Suzuka Nat. Coll. Tech.)
The combination of biological systems and semiconductor nanotechnology offers great potential for device applications, such as high throughput and highly sensitive bio-sensors. Here, we have succeeded in fabricating a microwell array sealed with lipid bilayers on Si substrate for analysis of ion channel activity. In order to improve the stability of lipid bilayers suspended over microwells, an overhang shape at the microwell aperture was formed. Therefore, the lipid membrane was prevented from falling into the microwells, and fluorescent probes could be confined for more than one hour. A lipid membrane formed by rupturing a giant unilamellar vesicle is guaranteed to be a single lipid bilayer and is free from any organic solvents, making it the ideal environment for the study of membrane proteins.

Using the microwell array, $\mathrm{Ca}^{2+}$ ion transport through channels formed by $\alpha$ hemolysin was investigated by the change in the intensity of the $\mathrm{Ca}^{2+}$-sensitive fluorescent probe (fluo-4). After forming the $\mathrm{Ca}^{2+}$ ion gradient between the inside and outside of the microwells across the lipid bilayer, $\alpha$-hemolysin was introduced. The fluorescence intensity of fluo- 4 in the microwells was increased by $\mathrm{Ca}^{2+}$ ion transport. The small microwell volume allows for very sensitive detection of ion transport. We demonstrate that the microwell array with confined fluorescent probes sealed is a powerful tool for the functional analysis of ion channel-type membrane protein.

\section{A1346 On-chip analyses of the function of ATP-binding cassette (ABC) transporters}

Hirotaka Sasaki ${ }^{1}$, Ryuji Kawano ${ }^{1}$, Toshihisa Osaki ${ }^{1}$, Koki Kamiya ${ }^{1}$, Shoji Takeuch ${ }^{1,2}$ ( ${ }^{1}$ Kanagawa Academy of Science \& Technology, ${ }^{2}$ Institute of Industrial Science, The University of Tokyo)

ATP-binding cassette $(\mathrm{ABC})$ transporters, a protein superfamily that ranges from prokaryotes to eukaryotes, transport a wide variety of substrates across membranes, including metabolic products, lipids, and drugs. They utilize the energy of ATP hydrolysis for transport, and are involved in various human diseases, such as cancer and cystic fibrosis. Much attention has been paid in recent years to $\mathrm{ABC}$-transporters as potential drug targets, and high-throughput drug-screening systems for $\mathrm{ABC}$-transporters are in great demand. Although we have succeeded in detecting ATP-dependent transport by the immobilized ABCtransporters in polydimethylsiloxane (PDMS) microfluidic channels, relatively strong background fluorescence originated from vesicle-bound substrates was a problem for quantitative analyses of the interaction between $\mathrm{ABC}$-transporters and drugs. We overcame this issue by using a very low concentration of fluorescent substrates for incubation. At this concentration, fluorescence originated from a trace amount of vesicle-bound substrates was too weak to be detected. In contrast, distinguishable levels of fluorescence appeared as the influx of fluorescent substrates progressed. Taking advantage of this procedure, we here present a first report of the on-chip accurate analyses of the inhibition of $\mathrm{ABC}$-transporters with several drugs, and single vesicle transport analyses representing the presence of functionally heterogeneous transporters in vesicles.

3A1358 Light induced ion conductive change in gel films which contain amino acids joined on a hydrogenated amorphous silicon film

Takaaki Ichikawa ${ }^{1}$, Takuya Takahashi $^{1}$, Yuki Hiramitsu ${ }^{1}$, Yoshiyuki Nomoto ${ }^{1}$, Hiroshi Masumoto ${ }^{2}$, Takashi Goto ${ }^{3}$, Yutaka Tsujiuchi ${ }^{1}\left({ }^{1}\right.$ Materials Science and Engineering, Akita University, ${ }^{2}$ Center for Interdisciplinary Research, Tohoku University, ${ }^{3}$ Institute for Materials Research, Tohoku University)

Recently, hydro-gel has been played a very important role in gel pad or bio electro chemical device. In the case of electrical defibrillation, large amount of electricity is poured into human body, in very short time, i.e., in some milliseconds. On the other hand, in the case of electro chemical device in human body or on human skin, in the course of long period the organ exposes itself to ion conductive action of the gel pad. Therefore, it is desirable to construct a gel pad using highly safe materials. Amino acid is the elementally element of bio molecule, has potential of diversity to electro chemical device. Our group has been working with amino acids dispersed in agarose gel, and with semiconductive material, hydrogenated amorphous silicon (a-Si: H) film. We measured recently ion conductive current in laminated two agarose gels that contain different amino acid. In this report, using Cottrell equation, ion conductive behavior of neutral, acidic, or basic amino acid dispersed in agarose gel are characterized. An appropriate combination of amino acid, to construct a gel that can produce large current with rectification property and to be capable of producing a tailor made designed gel pad with current strength selectivity, are discussed. Furthermore, we report an attempt of measuring a light induced effect of a-Si: $\mathrm{H}$ film on ion conducting behavior, comparing several hydrogen concentrations that used in process gas for preparation of a-Si:H. 